Revue des patrimoines

$26 \mid 2015$

La reconversion des sites et des bâtiments industriels

\title{
La reconversion de la centrale Montemartini dans le quartier d'Ostiense à Rome
}

Géraud Buffa

\section{OpenEdition}

Journals

Édition électronique

URL : http://journals.openedition.org/insitu/11782

DOI : $10.4000 /$ insitu. 11782

ISSN : 1630-7305

Éditeur

Ministère de la Culture

Référence électronique

Géraud Buffa, "La reconversion de la centrale Montemartini dans le quartier d'Ostiense à Rome », In Situ [En ligne], 26 | 2015, mis en ligne le 06 juillet 2015, consulté le 26 mars 2020. URL : http:// journals.openedition.org/insitu/11782 ; DOI : https://doi.org/10.4000/insitu.11782

Ce document a été généré automatiquement le 26 mars 2020

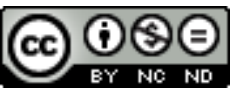

In Situ Revues des patrimoines est mis à disposition selon les termes de la licence Creative Commons Attribution - Pas d'Utilisation Commerciale - Pas de Modification 4.0 International. 


\title{
La reconversion de la centrale Montemartini dans le quartier d'Ostiense à Rome
}

\author{
Géraud Buffa
}

Rome a fêté en 2012 le centenaire de l'inauguration de la centrale Montemartini, qui fut la première centrale électrique publique de la Ville. Cent ans après sa construction, cette centrale présente aujourd'hui un visage fort différent de celui qu'elle avait alors. Tout d'abord parce que son existence a été ponctuée de profondes transformations et d'agrandissements au cours desquels sa puissance fut notablement augmentée. Ensuite et surtout, parce que depuis sa fermeture en 1968, elle a déjà connu deux reconversions successives en lieux culturels. Si la première ne fut pas un grand succès, force est de reconnaitre que la seconde s'est d'emblée propulsée au rang des grandes réussites, de celles qui soulève l'enthousiasme d'un public nombreux et varié (fig. 1). 
Figure 1

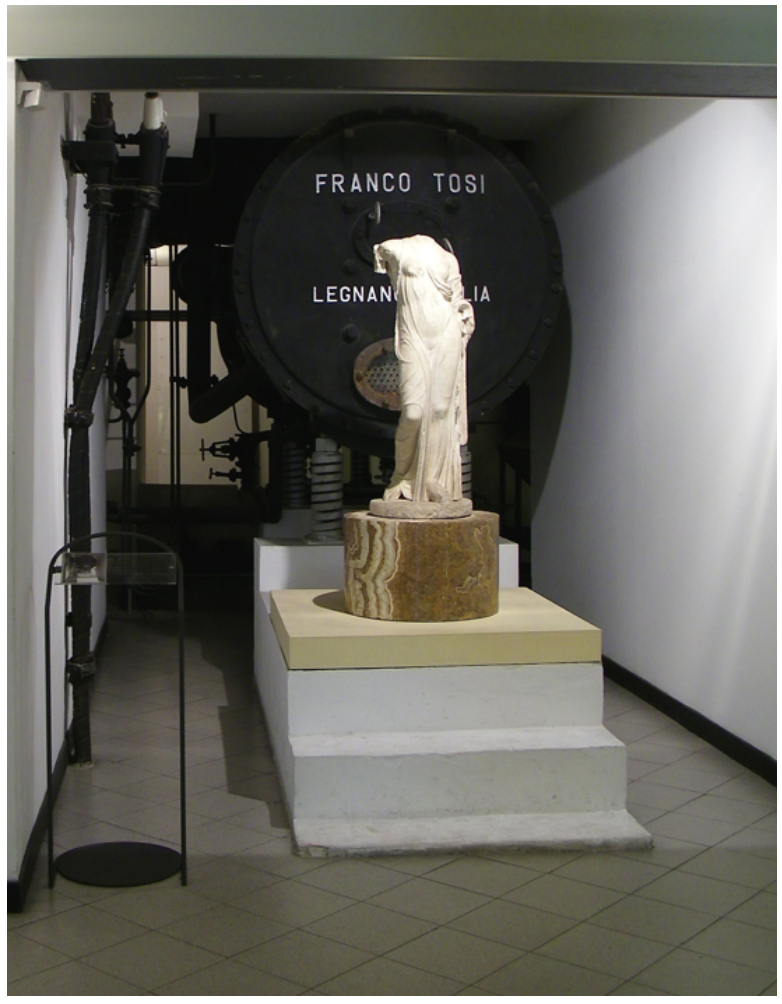

Une vue du hall d'entrée.

Phot. Buffa, Géraud, 2011. @ Géraud Buffa.

\section{Aux origines de la centrale}

2 Jusqu'en 1912, la Ville de Rome tirait exclusivement son électricité de centrales concédées à une compagnie privée, la Société anglo-romaine du gaz (SAR). Dès 1906, elle avait envisagé la création d'une centrale électrique publique utilisant la vapeur mais ce projet avait cheminé assez lentement du fait des enjeux politiques qu'il comportait. La production d'électricité par une entreprise publique était en effet une des revendications de l'opposition socialiste et trouvait assez peu d'écho auprès de la majorité dominée par l'organisation cléricale Unione Romana. Après la victoire électorale du parti progressiste Blocco Popolare en juin 1907, la production d'électricité par les services municipaux devint une priorité. La précédente majorité avait eu le temps de décider que la nouvelle usine serait bâtie juste à côté d'une usine à gaz construite par la SAR, sur un terrain de $20000 \mathrm{~m}^{2}$ situé au sud de Testaccio, entre le Tibre et la via Ostiense, non loin de la basilique Saint-Paul-hors-les-Murs (fig. 2). Installer l'usine sur les bords du fleuve offrait la possibilité d'approvisionner facilement en eau et en charbon les chaudières de la future centrale. Le projet connut alors une accélération décisive sous l'impulsion du conseiller municipal Giovanni Montemartini, ce qui explique que son nom ait été donné à la centrale au moment de son décès en 1914. Peu avant le début du chantier, la capacité de la future centrale fut doublée et passa à $7500 \mathrm{~kW}$. La décision fut également prise de lui adjoindre une centrale hydroélectrique chargée de garantir la fourniture du courant pendant les périodes de pointe. Ce changement d'échelle dans le projet fut soumis à la population romaine qui 
l'approuva à une écrasante majorité en septembre 1909 lors du premier référendum de l'histoire de la ville. Cette même année fut créée l'Azienda Elettrica Municipale, Comune di Roma (AEM) pour exploiter la centrale et assurer la distribution de l'électricité1.

Figure 2

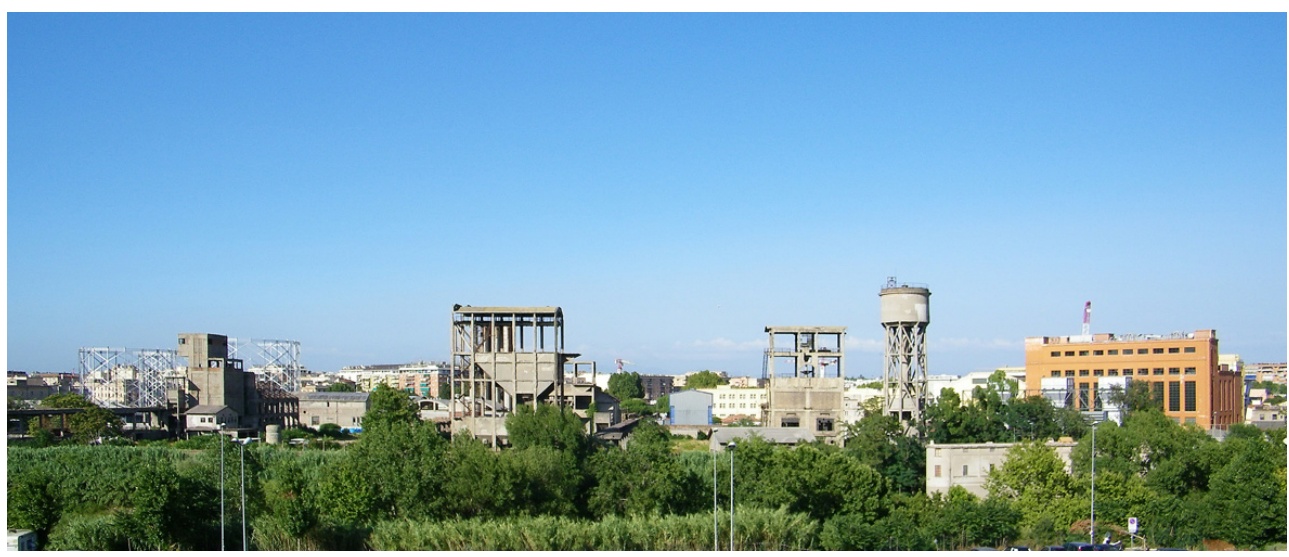

Les bords du Tibre vus de la rive droite. À gauche les vestiges de l'usine à gaz ; à droite la centrale Montemartini.

Phot. Buffa, Géraud, 2011. (c) Géraud Buffa.

Une seconde modification importante fut apportée au projet initial. Des moteurs diesel furent substitués aux turbo-alternateurs à vapeur initialement envisagés. La technologie diesel, alors en plein essor, semblait mieux à même de fournir à moindre coût la quantité d'électricité demandée. L'investissement initial était en effet inférieur puisqu'il rendait caduque la construction des énormes chaudières nécessaires à la production de la vapeur et les moteurs diesel devaient aussi réduire les coûts du fonctionnement de l'usine puisqu'ils réclamaient moins de main-d'œuvre au quotidien. Le marché de construction de ces moteurs fut attribué à l'entreprise Franco Tosi de Legnano qui s'engagea à fournir le matériel pour la fin de l'année 1912 et la première pierre de la centrale fut posée en présence de Victor-Emmanuel III le 25 janvier 1911.

Tosi ne put tenir les délais. Pour assurer l'ouverture de la centrale à la date prévue, une nouvelle modification du projet fut décidée. Aux moteurs diesel fut finalement adjointe en toute urgence l'unité de production électrique fonctionnant à la vapeur que Tosi exposait alors à l'Exposition universelle de Turin. Ces modifications entraînèrent la construction, à côté de la salle des machines principales, de nouveaux bâtiments pour abriter les chaudières.

\section{Un demi-siècle d'activité industrielle}

Lors de son inauguration, le 30 juin 1912, la première centrale publique de production d'électricité de Rome était donc une centrale mixte utilisant deux techniques très récentes et promises à un bel avenir : le moteur diesel et le turbo-alternateur. Mais les moteurs diesel, malgré leur taille imposante, ne fournissaient qu'une petite part de l'électricité produite. Les turbines à vapeur constituaient en effet l'essentiel de cette production. Le chantier de la centrale avait donc dû une fois de plus s'adapter pour que soient mises en place les installations nécessaires au retour massif que la vapeur faisait 
dans le projet. Ce retour fut d'autant plus important que c'est encore la vapeur qui fut choisie en 1917 et 1924 pour augmenter la capacité de la station (fig. 3).

Figure 3

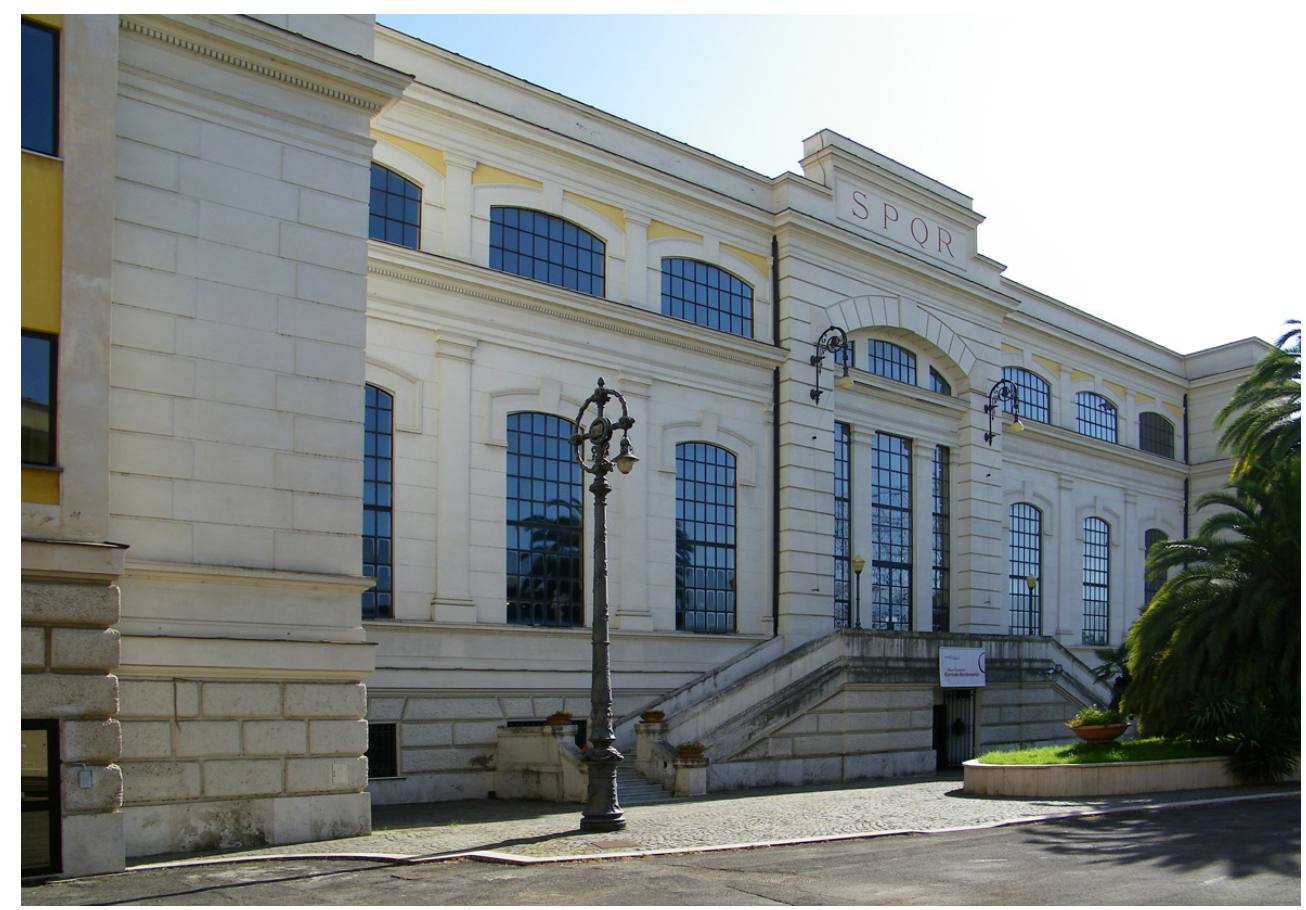

La façade de l'usine (élévations nord).

Phot. Buffa, Géraud, 2011. @ Géraud Buffa.

6 La capacité de l'usine utilisant la technique du diesel fut à son tour améliorée en 1933. Pour faire face à l'augmentation continue des besoins en électricité, les moteurs d'origine furent remplacés par deux nouveaux modèles, près de quatre fois plus puissants que les précédents et pour le coup véritablement gigantesques. Chacun de ces deux moteurs diesel à deux temps mesurait près de $20 \mathrm{~m}$ de long pour $7 \mathrm{~m}$ de hauteur et presqu'autant de largeur (fig. 4). Ces moteurs, toujours fournis par la firme Tosi, sont ceux qui sont encore présents dans l'usine. À cette occasion la centrale fut inaugurée une seconde fois, et par la même occasion, le régime fasciste décida de la rebaptiser pour faire disparaître le souvenir politique associé à Montemartini. 
Figure 4

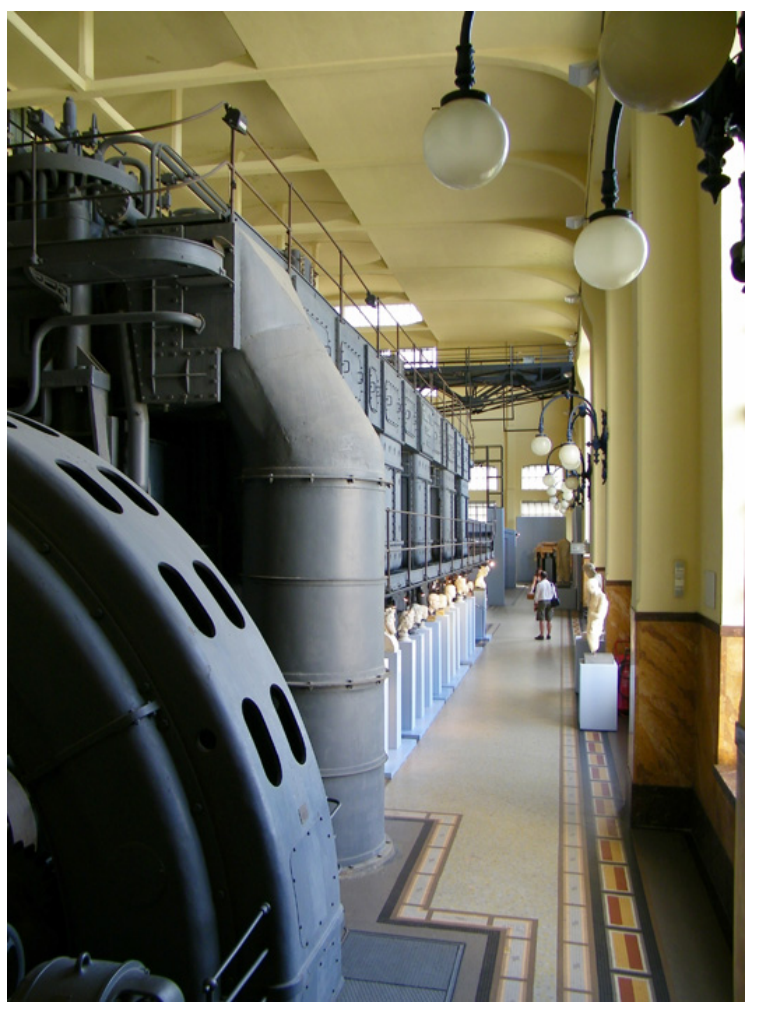

Vue latérale d'un des deux moteurs installés en 1933.

Phot. Buffa, Géraud, 2011. (c) Géraud Buffa.

7 Une nouvelle modernisation importante de la centrale fut entreprise dans les années 1940, avec le remplacement des chaudières à vapeur. Un vaste bâtiment fut construit pour les accueillir (fig. 5). Par la suite, l'aspect de l'usine ne fut plus vraiment modifié. La centrale eut en effet la chance de ne pas être gravement touchée par les bombardements aériens de la Seconde Guerre mondiale. La dernière transformation significative concerna le nouveau bâtiment qui fut agrandi vers 1950 pour l'installation d'une troisième chaudière, celle qui est encore en place. 
Figure 5

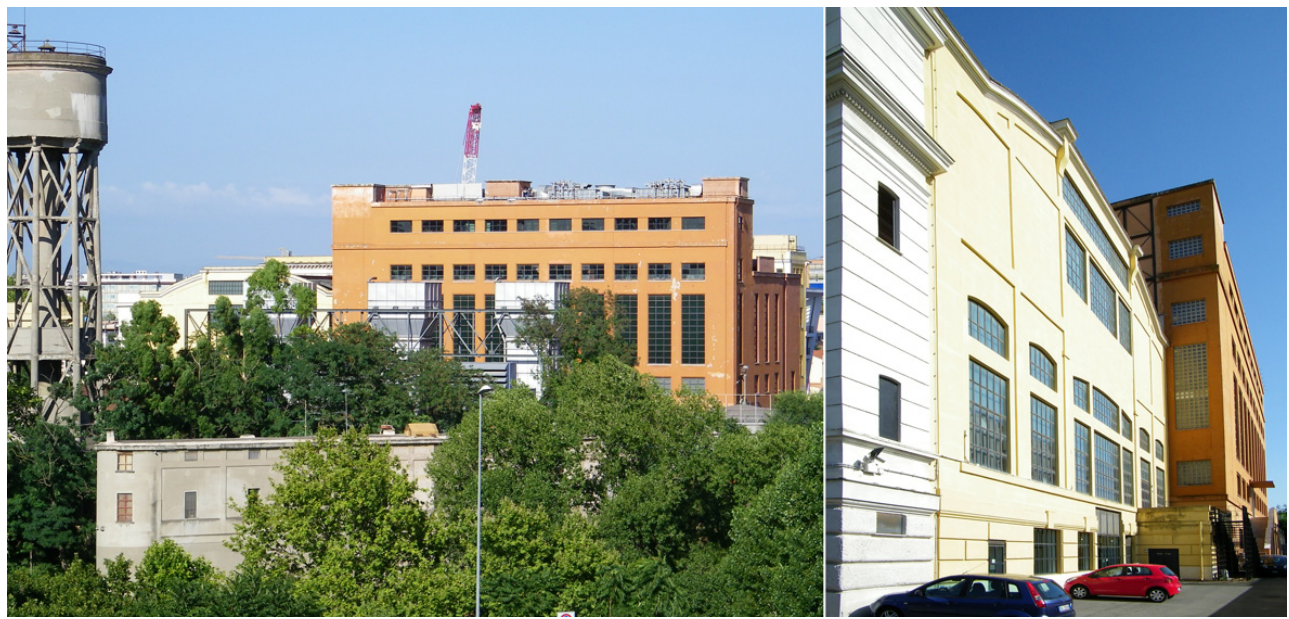

Élévations ouest de la centrale. À gauche la salle des machines; à droite (de couleur orange) le bâtiment construit pour l'installation des nouvelles chaudières dans les années 1940.

Phot. Buffa, Géraud, 2011. (c) Géraud Buffa.

Le déclin de l'usine fut par la suite assez rapide et son exploitation cessa définitivement à la fin des années 1960. L'usine servit alors d'entrepôt et de bureaux à l'ACEA (Azienda comunale Energia e Ambiente). Vingt ans plus tard, elle fit l'objet d'une première reconversion. En 1989 ouvrait le centre multimédia et centre d'art Acea, sorte de CCSTI ${ }^{2}$ dédié à l'archéologie industrielle et à l'histoire de l'électricité. Cette transformation ne fut malheureusement ni un succès public, ni une réussite sur le plan de la conservation du patrimoine puisqu'à cette occasion, une part non négligeable des installations de la centrale fut détruite, dont deux des plus anciennes des trois grandes chaudières Tosi installées dans les années 1940.

\section{"Les machines et les dieux »: les collections des musées du Capitole à la centrale Montemartini}

Six ans plus tard, en 1995, apparut l'idée qui allait conduire à l'état actuel de la centrale. La conservation des musées de Rome souhaita en effet l'utiliser comme lieu d'exposition temporaire pour accueillir pendant trois ans les collections des musées du Capitole qui devaient connaître une importante campagne de travaux.

Contrairement à ce qu'il est désormais courant d'observer lors de la reconversion de sites industriels, ce n'est donc pas l'art contemporain qui a été choisi pour réinvestir la centrale. C'est ici sans doute un des nombreux privilèges de la capitale italienne : ses ressources en œuvres d'art antiques sont extraordinaires.

11 Le nouveau lieu inauguré en 1999 était alors quasi révolutionnaire. L'usage n'avait pas encore rendu courante la présentation d'art contemporain dans les palais anciens et le public était peu habitué à voir dialoguer des artefacts produits par l'homme à des siècles de distance. L'exposition s'intitulait « Les machines et les dieux » ${ }^{3}$. Devant l'acier noir des immenses moteurs et des installations industrielles du siècle dernier venaient s'aligner d'antiques visages de marbre blanc aux harmonieuses proportions humaines (fig. 6, fig. 7). 
Figure 6

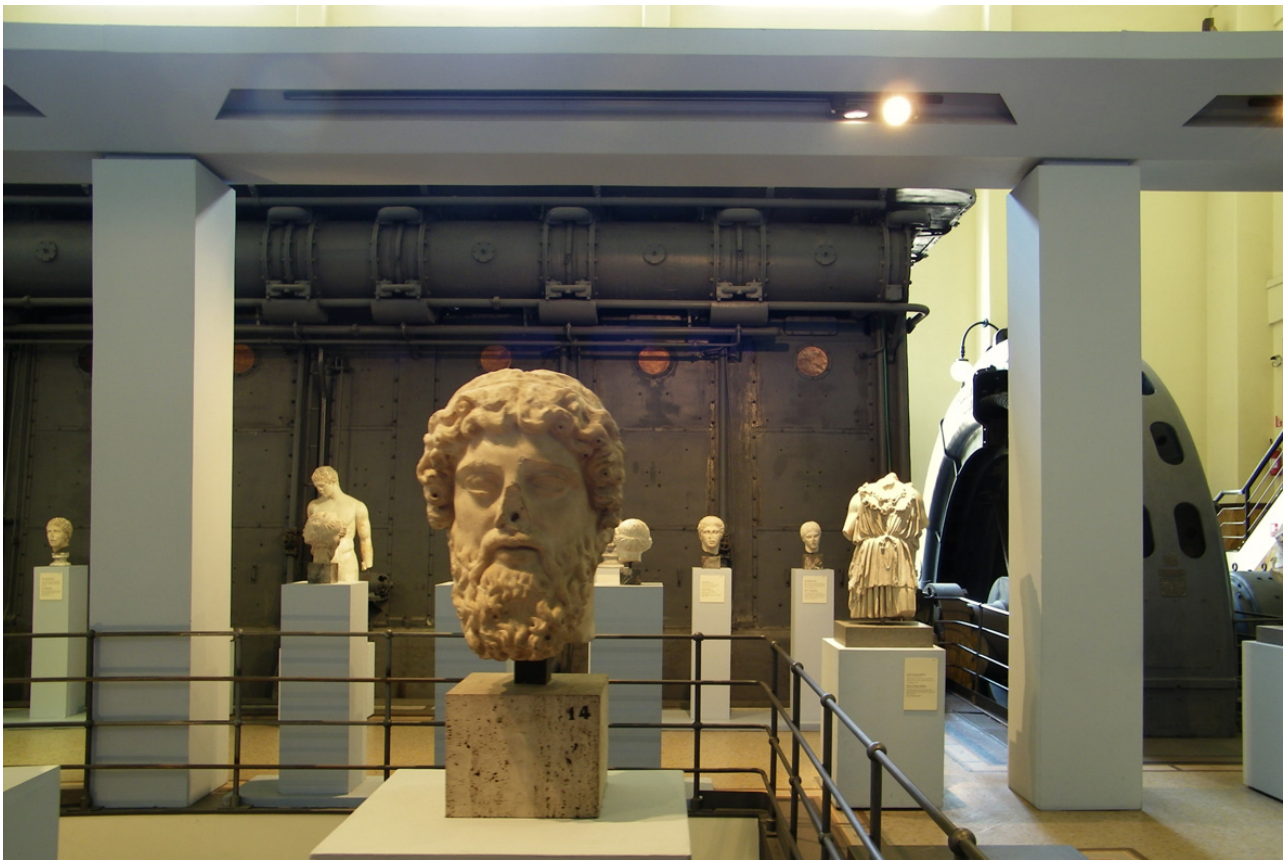

Exposition de marbres antiques dans la salle des machines.

Phot. Buffa, Géraud, 2011. @ Géraud Buffa.

Figure 7

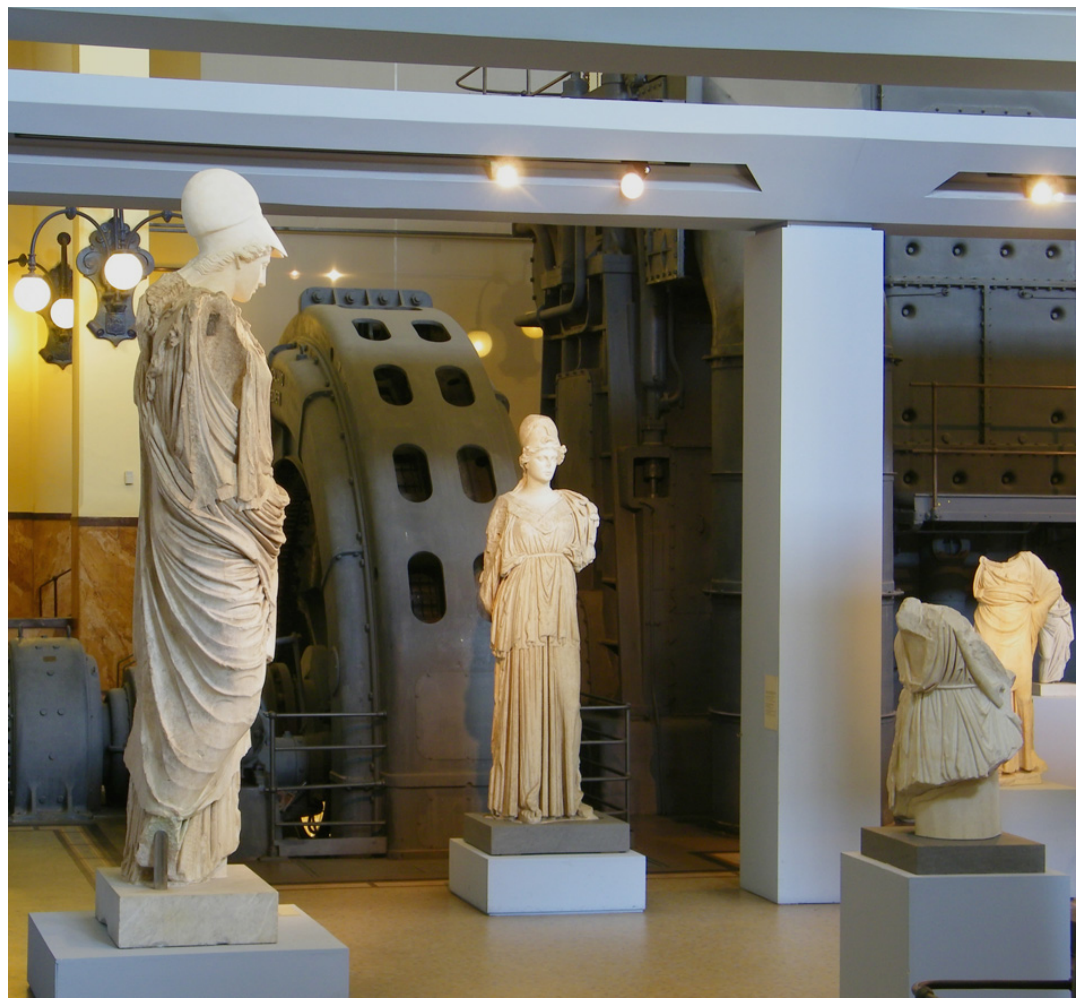

Exposition de marbres antiques dans la salle des machines.

Phot. Buffa, Géraud, 2011. ㄷ Géraud Buffa. 
12 provisoire : il permettait une continuité dans l'exposition des collections de sculpture. Sans être de courte durée, cette exposition devait donc être malgré tout temporaire, et ce statut intermédiaire autorisait toutes les audaces. L'audace a payé. Le succès, l'engouement même, fut immédiat, et le lieu fut assez vite à la mode. Il fut investi par le cinéma. Il fut choisi pour des concerts. La confrontation marchait à plein.

Il fut donc décidé de pérenniser le principe de cette exposition lorsque les travaux furent achevés au Capitole. En 2005, la centrale Montemartini devenait un des nouveaux sites de la conservation des musées archéologiques de la Ville de Rome, désormais consacré à la conservation et à la mise en valeur des acquisitions les plus récentes (issues des fouilles de la fin du XIX ${ }^{e}$ et du Xx ${ }^{e}$ siècle) des musées du Capitole.

Quatre espaces ont été définis pour structurer le parcours. Le hall d'entrée, situé sous la salle des machines, est entièrement dévolu à l'histoire de la centrale. Au même étage que l'accueil se trouve la salle dite des colonnes. Cet espace, très bas de plafond, est ainsi dénommé en raison de la forêt de piliers qui l'encombre et soutenait les lourdes chaudières du premier étage. On y trouve pour l'essentiel des sculptures illustrant les arts funéraires et la pratique du portrait.

Figure 8

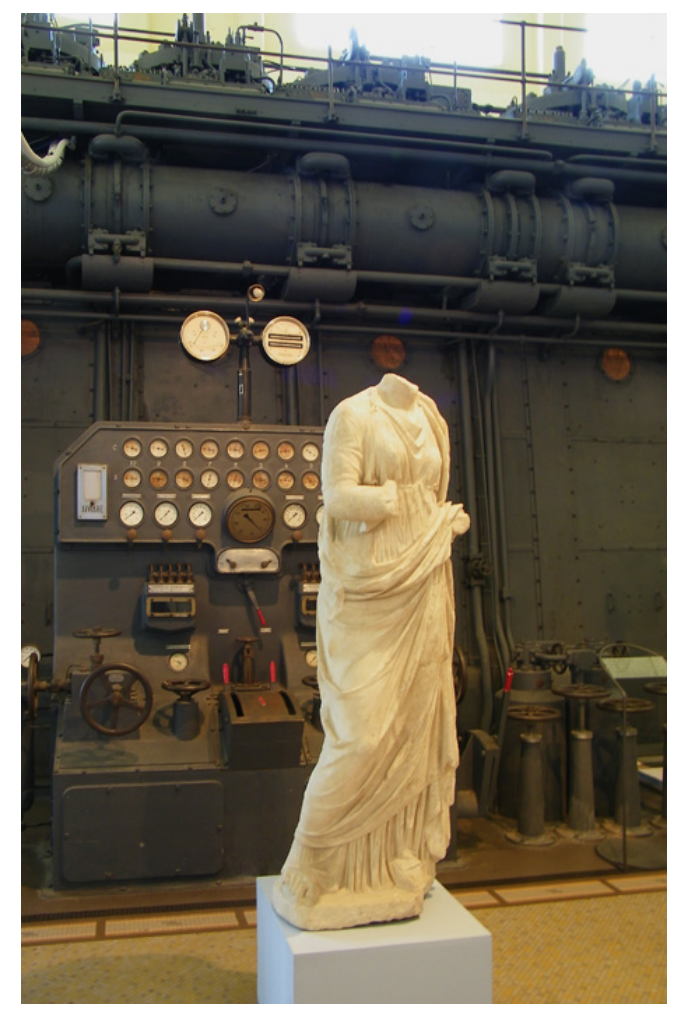

Statue d'Hygie devant le tableau de bord d'un des moteurs diesel.

Phot. Buffa, Géraud, 2011. @ Géraud Buffa.

Le premier étage a été divisé en deux espaces. Les collections antiques sont réparties en fonction des lieux où elles ont été découvertes. Dans la salle des machines, où se trouvent les deux moteurs de 1933, ont été déployés différents ensembles de sculptures grecques et romaines provenant de fouilles réalisées dans le centre de la Rome antique 
(fig. 8). C'est là qu'a été installée une reconstitution du fronton du temple d'Apollon Sosien édifié à proximité du théâtre de Marcellus (fig. 9). Dans la salle des chaudières sont regroupées des collections issues de fouilles pratiquées dans des zones plus périphériques à l'époque impériale (qui correspondent aujourd'hui à la gare centrale, la porte Saint-Laurent, Saint-Paul-hors-les-Murs...). On peut notamment y voir une grande mosaïque polychrome illustrant la capture de bêtes sauvages (fig. 10).

Figure 9

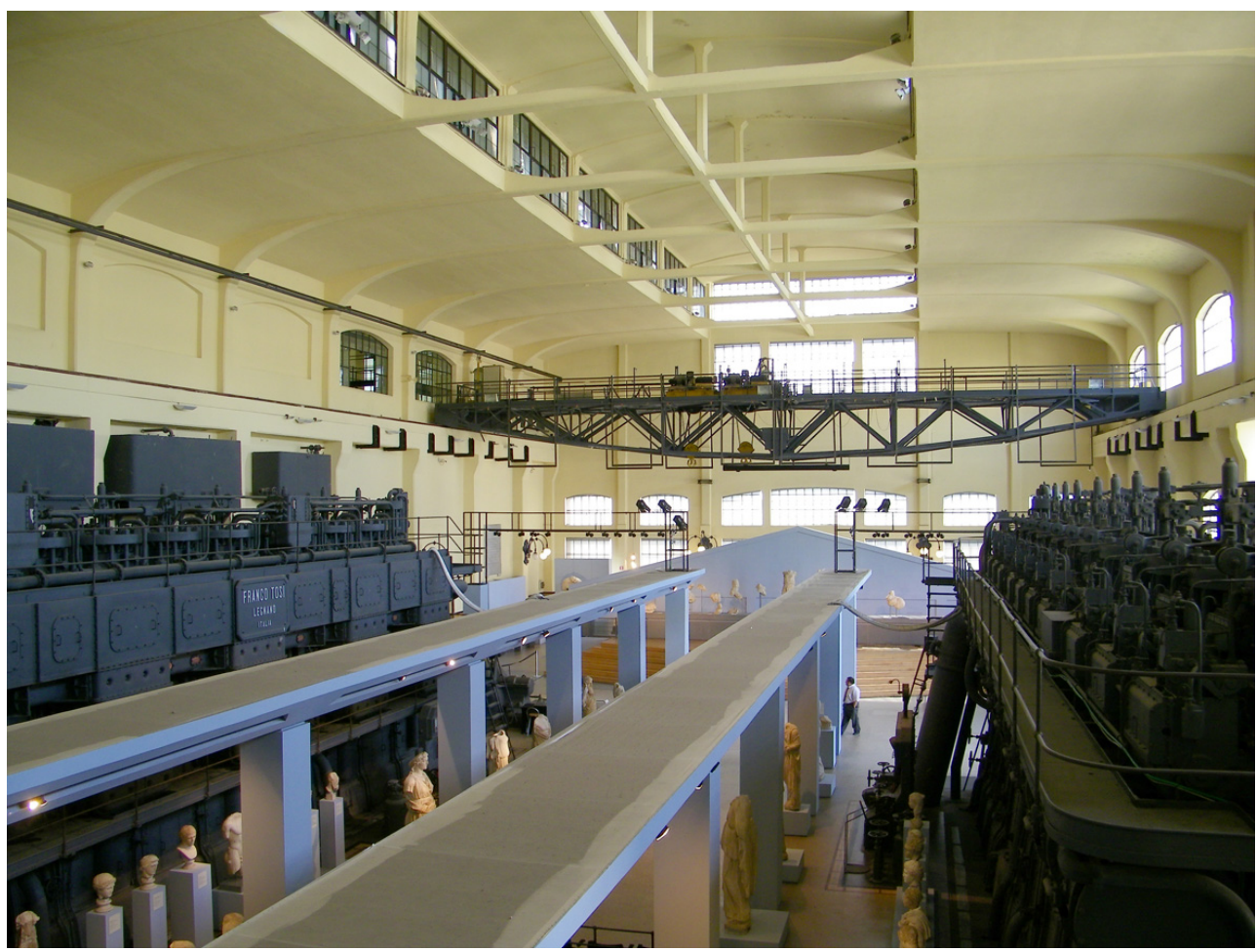

La salle des machines, vue générale. Au fond la reconstitution du fronton du temple d'Apollon Sosien. Phot. Buffa, Géraud, 2011. (c) Géraud Buffa. 
Figure 10

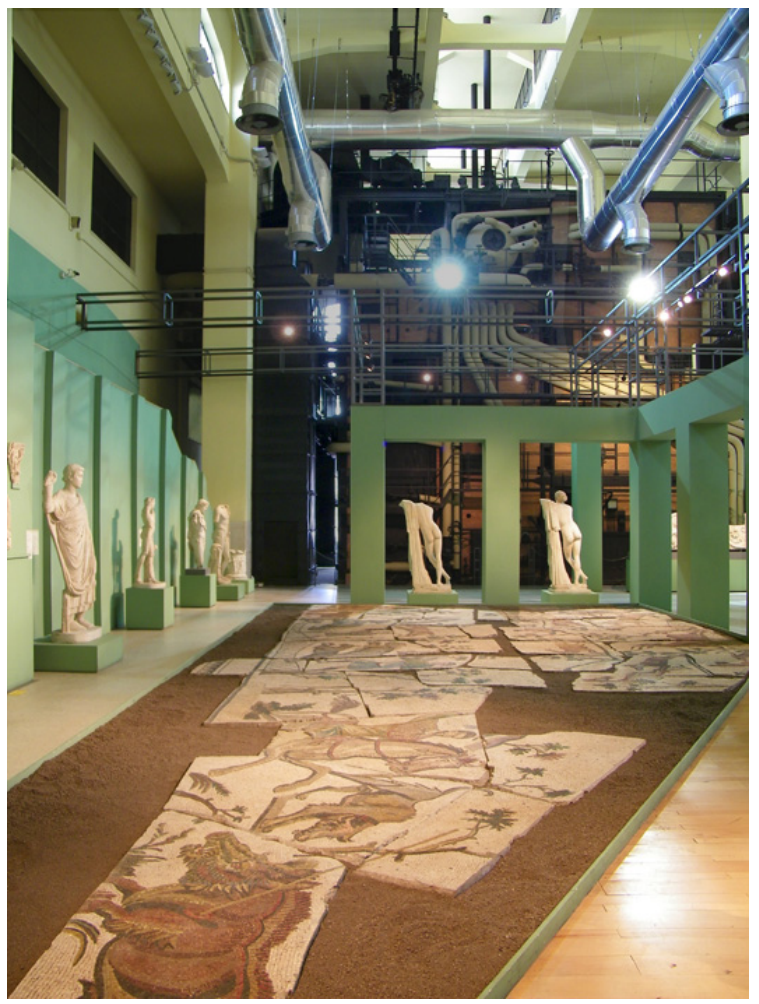

La salle des chaudières. Au fond la dernière chaudière Tosi de 1951 et au premier plan la mosaïque. Phot. Buffa, Géraud, 2011. (c) Géraud Buffa.

\section{Archéologie industrielle versus archéologie antique}

La force de cette exposition vient de ce qu'elle ne s'est pas simplement proposé de réutiliser un bâtiment: l'histoire de l'usine est longuement détaillée et les machines sont encore en place. Leur taille exceptionnelle interdit de les réduire à un simple décor. Elles s'imposent par elles-mêmes et il est impossible de porter le regard uniquement sur les sculptures ou uniquement sur les machines. Ce qui fait l'objet de cette exposition, c'est donc bien le dialogue entre " les machines et les dieux ».

Cette confrontation est si radicale qu'elle renouvelle immanquablement la compréhension de l'ensemble des objets proposés au visiteur, qu'ils relèvent de l'archéologie romaine ou de l'archéologie industrielle. Les esprits sont vite frappés par l'ellipse vertigineuse des siècles. Pour autant, le sens porté par cette exposition devenue permanente ne surgit pas comme une évidence. Cette difficulté tient probablement à ce qu'il n'est pas univoque. Car les contrastes sont si forts et les oppositions si nombreuses qu'ils se déclinent en une grande variété d'interrogations. Opposition physique des formes, des matériaux, des couleurs, des échelles, opposition des fonctions, opposition des époques, des esthétiques... Autant d'aspects qui expliquent que ce musée ne laisse personne indifférent. Ce concert de réactions, le plus souvent positives, suffit-il à justifier la démarche ? Il montre en tout cas que le souci de moderniser les manières de faire dans les musées peut être extrêmement profitable.

Si dans un premier temps on est frappé par la nouveauté de cette mise en scène, on s'aperçoit petit à petit qu'elle s'inscrit par bien des aspects dans la tradition des 
représentations du monde de l'industrie qui se sont imposées comme des icônes de notre temps. À ce titre, les questions d'échelle ne sont pas les moins intéressantes. La tension des corps des anciens dieux et la sérénité des visages parvenus jusqu'à nous à travers les siècles viennent souligner le gigantisme et la force du machinisme industriel tout aussi sûrement que la célèbre photographie du marteau-pilon de 125 tonnes de la Bethlehem Iron Company ${ }^{4}$. En retour, elles sont magnifiées, presque fragilisées, par le cadre de l'usine. En mettant face-à-face dans un même silence la figure de l'homme, ce qu'on peut voir en elle de plus universel, et la démesure des machines, ce musée prolonge la longue fascination des artistes qui, de Constantin Meunier ${ }^{5}$ à Lewis Heine ${ }^{6}$, ont mis en avant le corps de l'ouvrier au travail dans un cadre qui le dépasse et qu'il maitrise tout à la fois. En quelque sorte, elle transpose cette tradition iconographique dans le contexte plus traditionnellement prestigieux de l'histoire de l'art ancien. Si on prend le temps d'observer que les bustes sont alignés comme des rangs de soldats, que les courbes musculeuses des dieux semblent s'affronter à celles de l'acier, on se met vite à voir dans cette mise en scène une nouvelle représentation du combat de l'homme et de la machine (fig. 11, fig. 12).

Figure 11

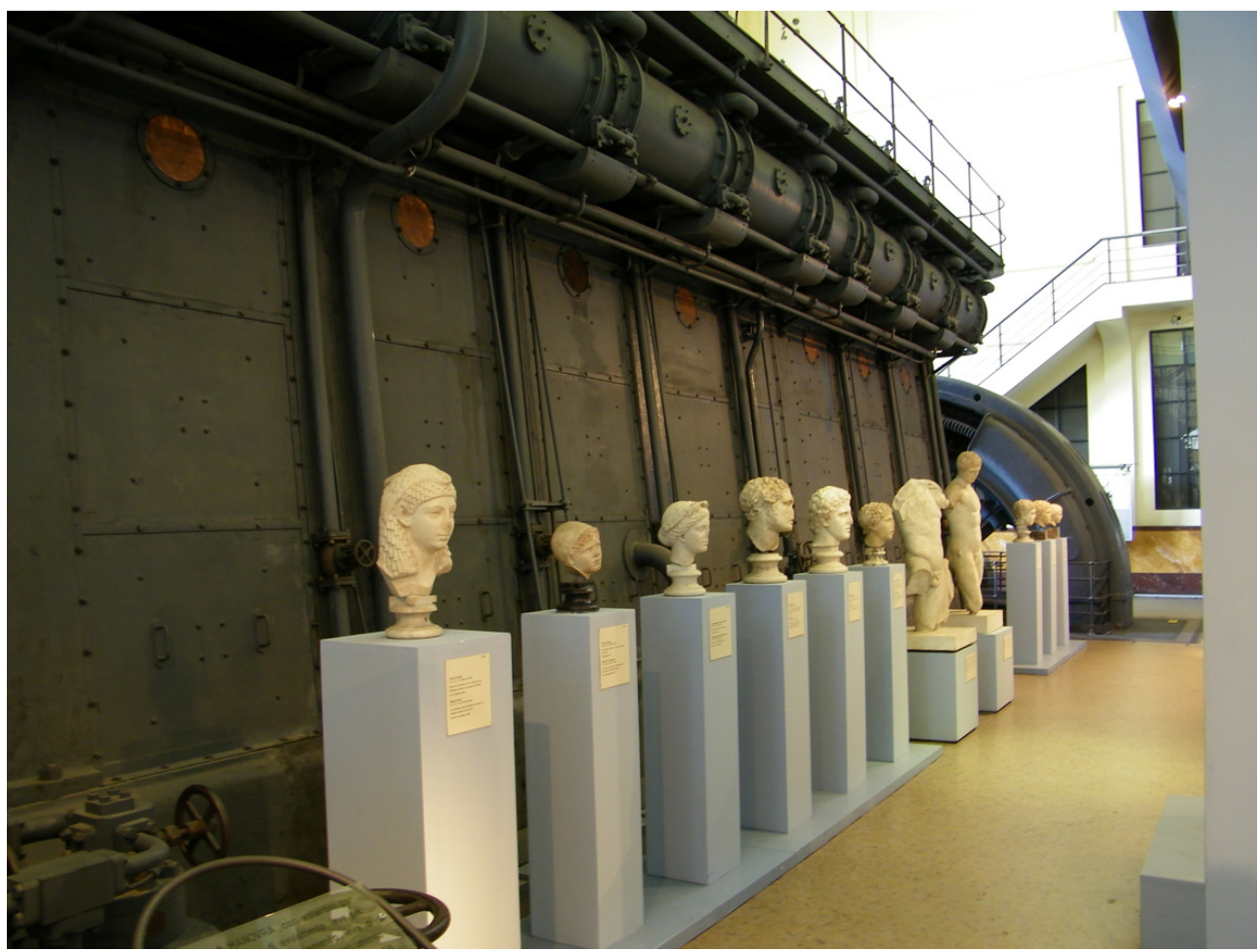

Alignement de têtes sculptées devant un des moteurs diesel.

Phot. Buffa, Géraud, 2011. (c) Géraud Buffa. 
Figure 12

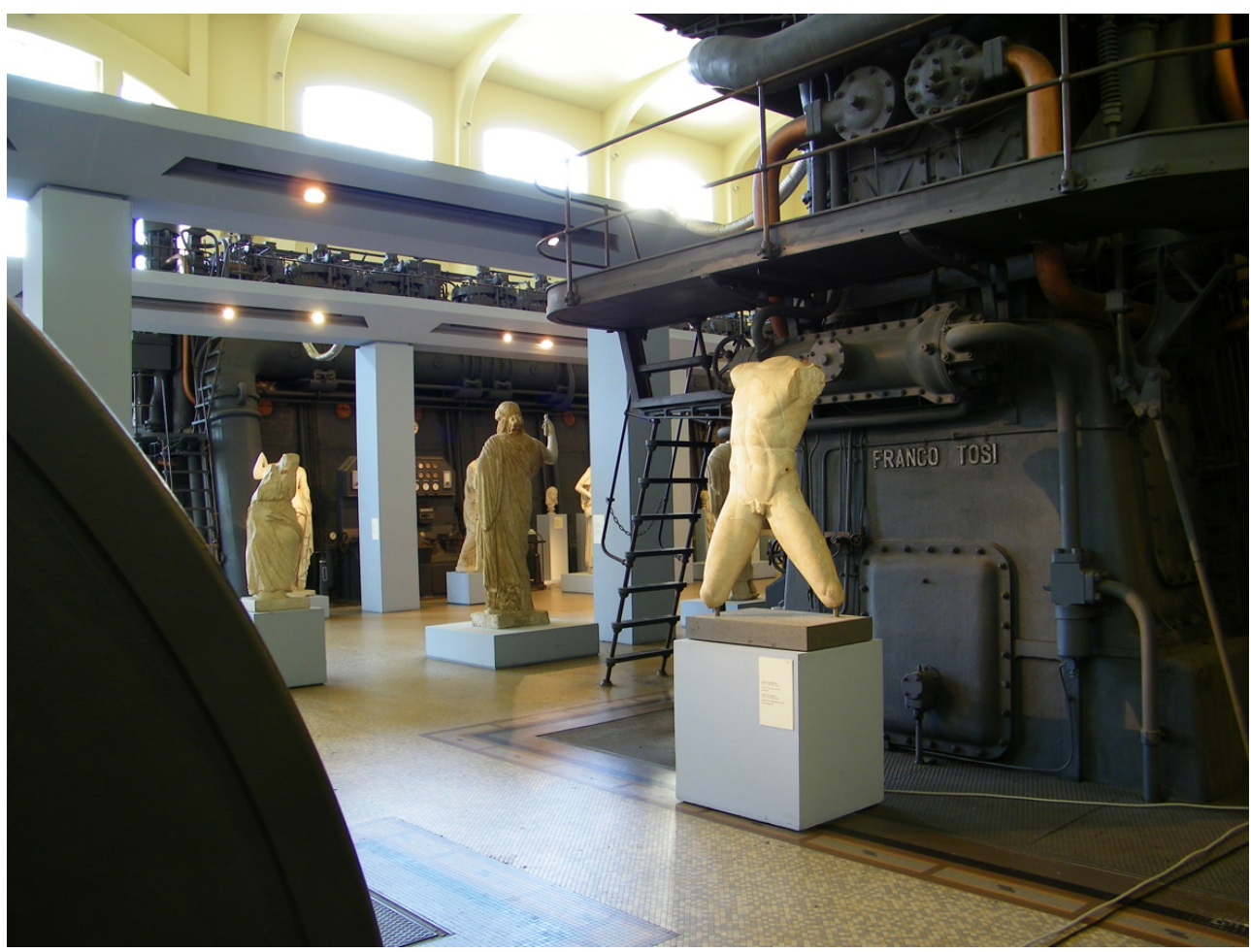

Torse de combattant devant un des moteurs diesel.

Phot. Buffa, Géraud, 2011. (c) Géraud Buffa.

Il ne faut pas beaucoup d'imagination pour y voir un rappel des conditions de travail des ouvriers qui se sont succédé dans l'entretien de l'usine. Car le visiteur a pu voir, dans la première partie de l'exposition, des photographies d'époque les montrant postés devant les tableaux de bord ou alimentant les chaudières, à l'endroit même où sont aujourd'hui exposées les œuvres d'art. La substitution de ces corps sculptés à ceux des mécaniciens est saisissante. Elle constitue un fort bel hommage au travail ouvrier en le resituant dans la perspective millénaire de l'homme qui réinvente sa propre condition. Dans un contexte où l'on reproche souvent aux reconversions de sites industriels d'effacer la partie humaine de la vie d'un lieu pour ne garder qu'une enveloppe dans laquelle le sens historique se perd, ce n'est pas un petit mérite (fig. 13). 
Figure 13

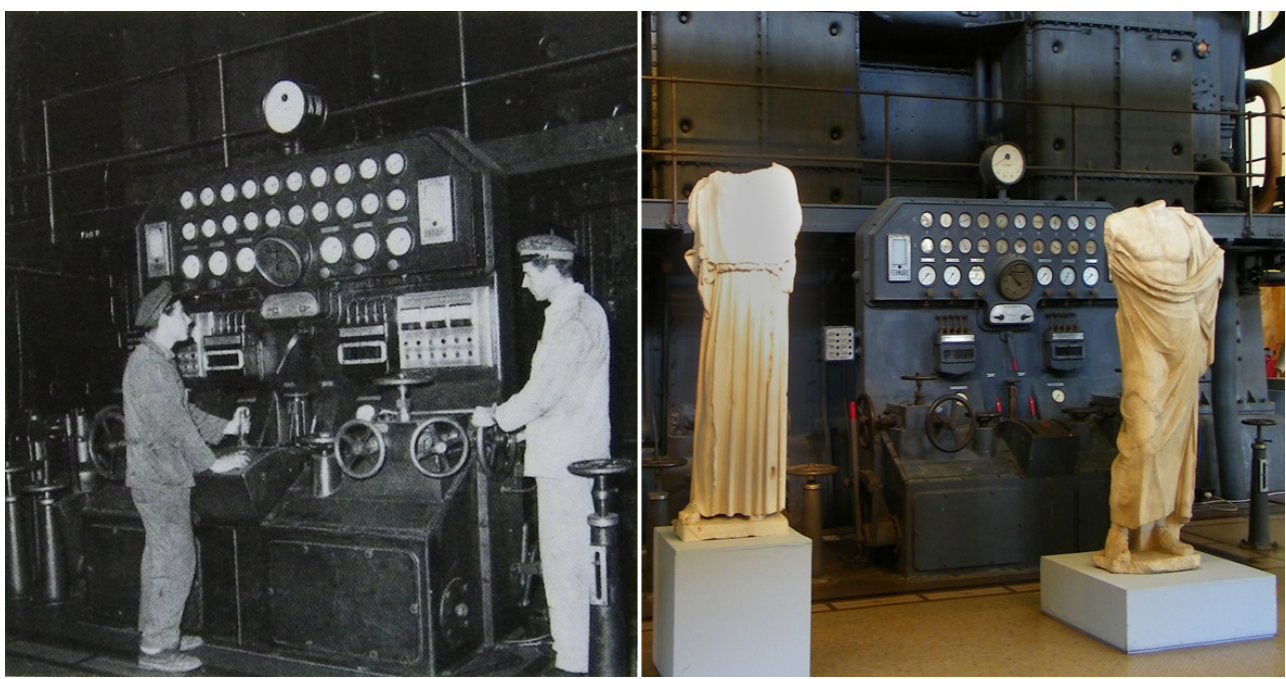

Ouvriers dans les années 1960 (photographie tirée de : BERTOLETTI M., TALAMO, E., CIMA M. (sous la direction de). Sculture di Roma antica. Roma : Electa, 2006, 139 p.) et sculptures antiques en 2011.

Phot. Buffa, Géraud. (c) Géraud Buffa. nourrit la réflexion sur la propension des objets du patrimoine industriel à évoquer la modernité, même lorsqu'ils sont les témoins, si ce n'est d'un système technique, du moins d'un machinisme dépassé. Les moteurs et la chaudière sont des objets de la première moitié $\mathrm{du} \mathrm{xx}^{\mathrm{e}}$ siècle, dont on ne saurait dire s'ils sont en général plutôt associés dans l'esprit de nos contemporains à de vénérables antiquités ou aux technologies les plus récentes, celles dont les ressorts sont désormais inaccessibles au grand public et qui ont envahi notre quotidien. La difficulté qu'un grand nombre de visiteurs éprouve immanquablement à comprendre ces machines malgré les explications de la première partie leur confère encore une aura de mystère. Et si la fée électricité fait sans doute moins rêver aujourd'hui qu'il y a un siècle, la réaction des touristes devant leur monumentalité n'est peut-être pas si différente de celle qu'aurait pu avoir un visiteur contemporain de leur fabrication. Dans ce contexte, la présence des collections des musées du Capitole n'est pas neutre et contribue elle aussi à les refaire basculer dans le champ de la modernité. Elle explique en tout cas qu'une des questions les plus communément soulevées par l'exposition concerne le statut des objets techniques contemporains dont on a parfois tendance à faire de nouvelles idoles.

Pour autant, tout n'est pas exempt de défaut dans cette aventure. Ce qui frappe le plus lorsqu'on s'intéresse à l'histoire de la création de ce musée, c'est bien l'absence d'explication claire sur les enjeux d'une telle confrontation entre l'archéologie romaine et l'archéologie industrielle ${ }^{7}$. Le visiteur est largement livré à lui-même et à ses impressions. Il est donc difficile de savoir avec certitude quels principes ont présidé à l'exposition juxtaposée de deux univers si différents, et chacun doit s'en remettre à sa propre conviction. Il semble que le hiatus chronologique et l'abysse qui sépare ces deux mondes aient suffi à justifier la démarche. En tout cas, il n'a pas paru nécessaire d'en donner des clés de lecture. On peut naturellement regretter ce parti-pris, qui peut conforter les détracteurs de la centrale en laissant penser qu'elle n'était que l'écrin original adapté à l'air du temps plus encore qu'aux collections antiques. Ce reproche serait naturellement très exagéré, car toute la première partie de l'exposition, dans 
laquelle la sculpture antique est peu présente, est consacrée à l'histoire de l'usine et à l'extension de l'électricité dans la ville de Rome. Mais ce qui peut surprendre, c'est bien la différence de traitement muséographique par le dispositif textuel (panneaux et cartels) entre cette première partie et les suivantes. Une approche plus cohérente aurait probablement fait naître le besoin de mieux mettre en avant les tenants et aboutissants de ce projet. On retombe ici sur la traditionnelle opposition entre, d'une part, les objets dont le statut d'œuvre d'art amène la médiation à se faire discrète et, de l'autre, les musées de société, plus enclins à intégrer les éléments didactiques à la scénographie. En fait, cette différence s'explique surtout parce que c'est à l'ACEA qu'a été confié le soin d'expliquer les machines, tandis que les musées du Capitole se concentraient sur leur cœur de métier : la conservation et la valorisation du patrimoine antique. Question de compétence sans doute. Mais dans un même musée, cette opposition ne pouvait-elle pas être quelque peu réduite ? Cela aurait pu inciter à ne pas trop exacerber la blancheur et le dépouillement des sculptures qui ont longtemps été mises en avant dans les musées archéologiques et que le contraste avec le noir des installations industrielles vient déjà renforcer.

On ajoutera que certains des panneaux dédiés aux machines sont à ce point démodés qu'on se demande si on ne les pas petitement récupérés du temps déjà lointain du centre multimédia et centre d'art. Le contraste avec la qualité de la muséographie environnante dessert le regard que le visiteur est amené à porter sur le patrimoine industriel. Ce beau lieu reste donc encore perfectible.

On le voit, ce dialogue entre le marbre froid et pourtant si humain d'une part et, de l'autre, l'acier autrefois propulsé par les lois de la thermodynamique, modifie le regard que le visiteur aurait porté sur des machines similaires dans un autre contexte. Ce faceà-face souligne ce que la machine nous dit sur le génie humain qui l'a conçue. Il lui transmet également une part de vie tout en favorisant un jeu de questionnement qui se répercute sur les œuvres d'art elles-mêmes. Il est en effet assez ironique d'observer que ces marbres sculptés n'ont d'humain que leur forme et un souffle que des siècles d'étude et d'admiration ont surchargé de sens. Les machines, conçues pour un cycle de vie déterminé, étaient, elles, tout entières façonnées pour le mouvement dans des chaleurs extrêmes. Muséifiées parce que le musée est venu à elles, elles rejoignent aujourd'hui dans une éternité mutilée, immobile et silencieuse les visages de pierre produits par les ateliers de sculpture de l'Antiquité.

\section{Une reconversion au cœur d'un quartier en pleine mutation}

Il paraît impossible de décrire le renouveau de la centrale Montemartini sans aborder, même de manière succincte, celui de son environnement urbain. Car les quartiers de Testaccio, d'Ostiense et de Marconi connaissent depuis une vingtaine d'années une transformation profonde assez caractéristique des reconversions urbaines des anciens quartiers industriels délaissés. Cette partie de la ville avait été profondément bouleversée à la fin du $\mathrm{XIX}^{\mathrm{e}}$ siècle en devenant la principale zone industrielle de la capitale italienne. Cette vocation n'était pas véritablement nouvelle. Dès l'Antiquité, ces rivages du Tibre étaient marqués par l'activité de l'emporium, le port fluvial où arrivaient les marchandises en provenance d'Ostie. Le nom du Testaccio provient du reste du Monte Testaccio, colline artificielle constituée des débris accumulés par des 
siècles d'activités portuaires. Dans la seconde moitié du XIX ${ }^{e}$ siècle, et plus encore au début $d u x^{e}$ siècle, cet espace autour du fleuve s'est métamorphosé. Le passage du chemin de fer, notamment grâce au pont Saint-Paul qui franchit le Tibre à partir de 1907 et à la construction de gares, celle de Roma Trastevere sur la rive droite, celle d'Ostiense sur la rive gauche, celle de San Paolo plus au sud, en fait une zone d'activité essentielle pour la vie de la ville.

Dans les années 1970 et 1980, Ostiense offrait un visage de friche pauvre et désolée. La plupart des usines fermaient et le quartier tout entier se dégradait. Ces grands espaces à l'abandon ont par la suite rapidement attiré de nouvelles activités. La proximité du centre de Rome, la place disponible et l'exemple proposé par de nombreuses villes européennes allait contribuer à en faire un quartier brillamment rénové.

Le processus de réhabilitation s'est appuyé sur des bases très solides qui expliquent largement le succès qu'il rencontre. C'est en effet un quartier qui jouxte le centre historique et les sites les plus prestigieux de l'Antiquité romaine, au sud de l'Aventin. On y trouve un patrimoine architectural riche non seulement de son histoire sociale et du poids qu'il occupe dans la mémoire récente de la ville mais également des facilités de reconversion qui sont propres à la plupart des sites industriels (grands volumes, grandes surfaces, structures solides, espaces de circulations internes et externes très commodes, autant de caractéristiques adaptées aux modes de vie contemporains). Enfin, c'est aussi un patrimoine aux qualités architecturales formellement très abouties.

Ce processus s'est d'abord manifesté, comme souvent, par l'apparition de lieux nouveaux dédiés à la jeunesse (du petit bar à la discothèque aménagée dans les grands volumes des anciennes usines). Ces nouveaux usages, au succès d'autant plus fulgurant qu'ils se sont développés dans un premier temps dans des lieux alternatifs et pas toujours légaux, ne sont plus aujourd'hui prépondérants. Si Testaccio est encore aujourd'hui un quartier où sortir, il attire déjà moins la jeunesse avide de lieux nouveaux, c'est-à-dire de lieux un peu moins balisés et pris d'assaut par les touristes. Désormais, les grandes opérations de reconversion à proximité de la centrale sont beaucoup plus institutionnelles et s'inscrivent dans un paysage urbain en pleine gentrification (fig. 14). 
Figure 14

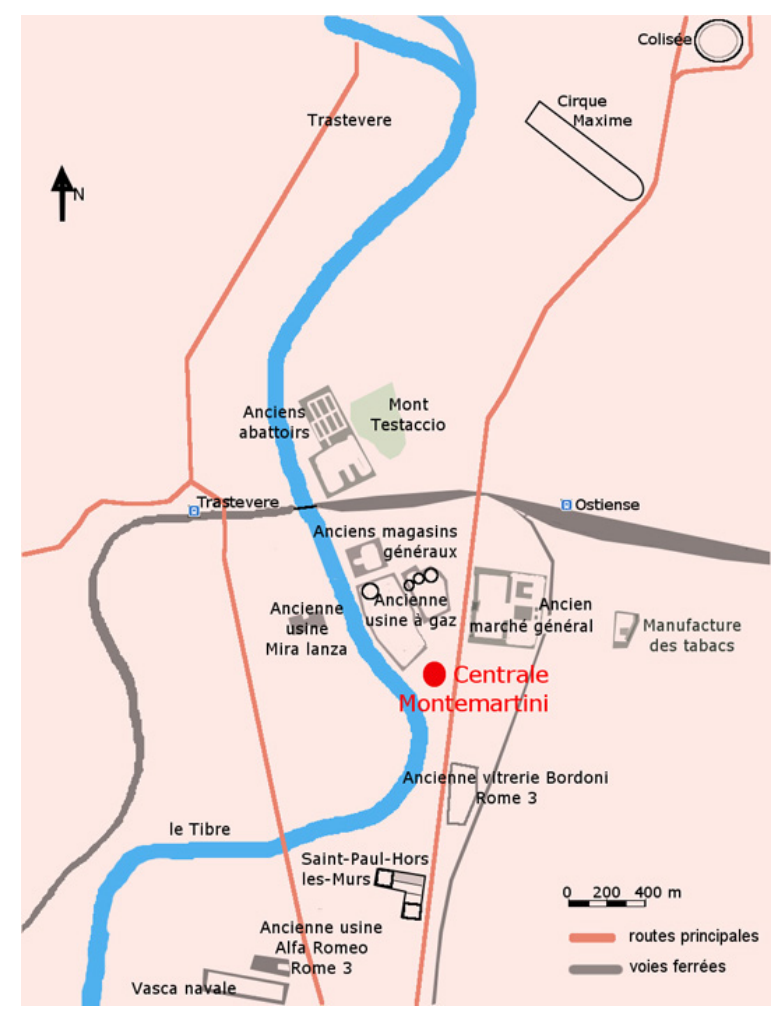

Plan de situation de la centrale et des différents éléments du patrimoine industriel reconvertis dans son voisinage.

Plan de l'auteur. (C) Géraud Buffa.

Une des plus importantes d'entre elles a investi depuis une quinzaine d'années le complexe des anciens abattoirs de Rome, fermés en 1975. Les anciens pavillons ont peu à peu été réutilisés par toute une variété d'organisations : la police municipale (2002), une annexe du musée d'art contemporain de Rome, le MACRO ${ }^{8}$ (2002), l'école d'architecture (2008) dépendant de la nouvelle université de Rome 3 et, plus récemment, des espaces conçus pour les industries de divertissement et de diffusion de la culture comme la Pelanda ${ }^{9}$ (2010). Et naturellement, on voit aujourd'hui fleurir tout autour de ces ensembles des programmes de logements avant-gardistes et hors de prix.

Le plus gros chantier actuellement en cours dans le voisinage de la centrale est conduit par l'agence de Rem Koolhaas. Il va métamorphoser l'immense site de l'ancien marché général de la ville, le long de la via Ostiense, où seront aménagés des lieux de divertissement, de restauration, de commerces, de bureaux, et des espaces dédiés à la culture.

Ces exemples de reconversion ont donc en commun d'être situés dans un périmètre réduit et de donner une nouvelle vie à des édifices industriels bâtis au tournant des XIx ${ }^{\mathrm{e}}$ et $\mathrm{xx}^{\mathrm{e}}$ siècles. Cette architecture industrielle est mise en avant pour ses qualités techniques et les facilités de réutilisation qui la caractérisent. Si ses matériaux sont assez variés et utilisent abondamment la pierre, le fer et le béton, les formes qui la composent le sont encore plus. Quelques sites, comme par exemple l'usine à gaz qui jouxte la centrale Montemartini, n'affichent dans leurs formes que leur fonction, sans souci de recherche architecturale. Cette usine à gaz fut construite par la SAR, la Société anglo-romaine du gaz, qui servait elle aussi à l'éclairage nocturne. Le plus haut de ses 
gazomètres s'élevant à près de $90 \mathrm{~m}$, il est visible en de nombreux points de Rome. Il est aujourd'hui un des éléments majeurs du paysage urbain de la capitale italienne (fig. 15)

Figure 15

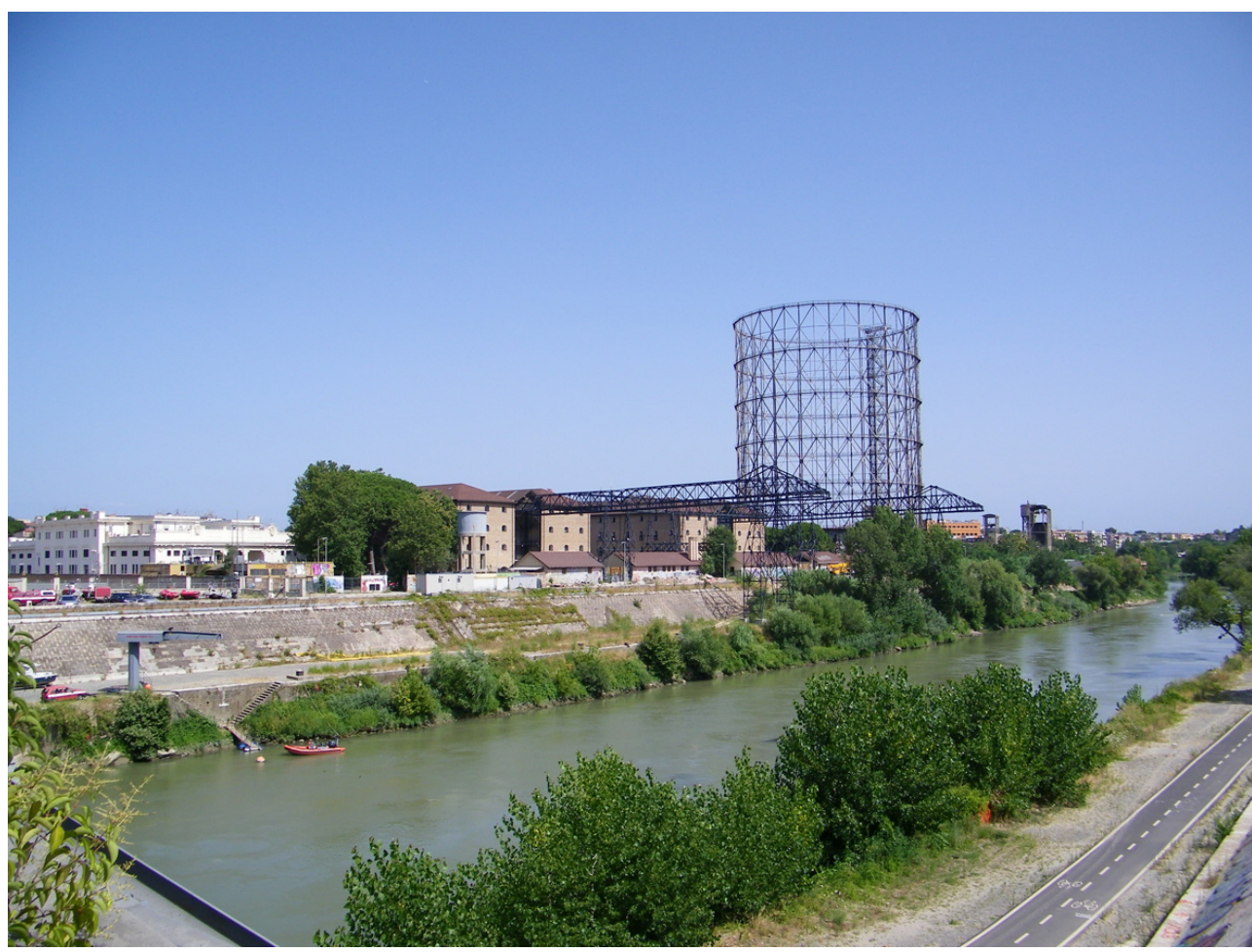

Les rives du Tibre vues du pont de l'industrie. De gauche à droite : les anciens magasins généraux, le gazomètre et au loin la centrale. À noter la récente piste cyclable aménagée en rive droite.

Phot. Buffa, Géraud, 2011. @ Géraud Buffa.

Mais le cas de cette usine, qui pourrait accueillir une part de la future Cité des sciences que projette depuis de nombreuses années la conservation des musées de la Ville, est une exception. La plupart des bâtiments industriels ont en effet développé toute une stratégie d'adaptation au cadre prestigieux de l'architecture romaine. On peut ainsi voir, parfois sur des distances hors norme, des façades dont la composition très soignée dissimulait les activités industrielles derrière les ordonnancements les plus savants et parfois les décors les plus soignés. La centrale électrique est à cet égard très significative. Ses grandes voûtes de béton sont difficiles à soupçonner lorsqu'on se trouve devant sa façade.

Tout près de la centrale, il faut encore mentionner les anciens magasins généraux qui accueillent depuis 2001 l'Istituto Superiore Antincendi (centre de formation des pompiers). Non loin également, de l'autre côté du fleuve, l'ancienne usine Mira Lanza Ostiense-Roma (fabrique de savon) a été transformée en théâtre contemporain, le Teatro India, et, à l'est du marché général, la manufacture des tabacs pourrait accueillir des bâtiments administratifs. Plus au sud, au 156 de la via Ostiense, se trouvait la vitrerie Bordoni (aujourd'hui détruite, à l'exception de l'entrée et d'un bâtiment de bureaux. Sur le site a été construite la faculté de droit de l'université de Rome 3). Un peu plus loin encore, autour de Saint-Paul-hors-les-Murs, sont reconvertis ou en passe de l'être la Vasca navale (construction navale), l'Ottica Meccanica Italiana (OMI, applications 
otiques pour le transport aérien et l'aérophotogrammétrie), la Società aerostatica Avorio (fabrication de parachutes), et encore plus au sud, au 234 de la via Ostiense, l'ancienne usine Alfa Romeo transformée pour abriter la faculté de lettres et philosophie de l'université Rome 3.

L'attention portée par la Ville de Rome à son plus ancien quartier industriel s'inscrit donc dans la droite ligne des grands travaux d'aménagements et de reconquête urbaine qui ont réussi à tant d'autres villes du Vieux Continent. Cette architecture offre ainsi aujourd'hui une nouvelle corde à l'arc patrimonial de la Ville de Rome qui n'est certes pas encore reconnue pour cet aspect de son histoire mais qui a compris tout le parti qu'elle pouvait retirer d'opérations prestigieuses où se combinent projets culturels, mise en valeur d'une architecture à laquelle l'image de la ville éternelle n'était jusquelà pas vraiment associée et grands noms de l'architecture internationale. Cette recette a fait ses preuves un peu partout, et Rome est actuellement en train de mettre en avant l'héritage industriel qui a façonné son visage actuel tout aussi sûrement que celui laissé par les empereurs ou les papes. La centrale Montemartini occupe dans ce nouveau paysage urbain une place toute particulière, qui a tous les atouts pour incarner le lien entre la période contemporaine et le passé prestigieux de la Rome antique (fig. 16).

Figure 16

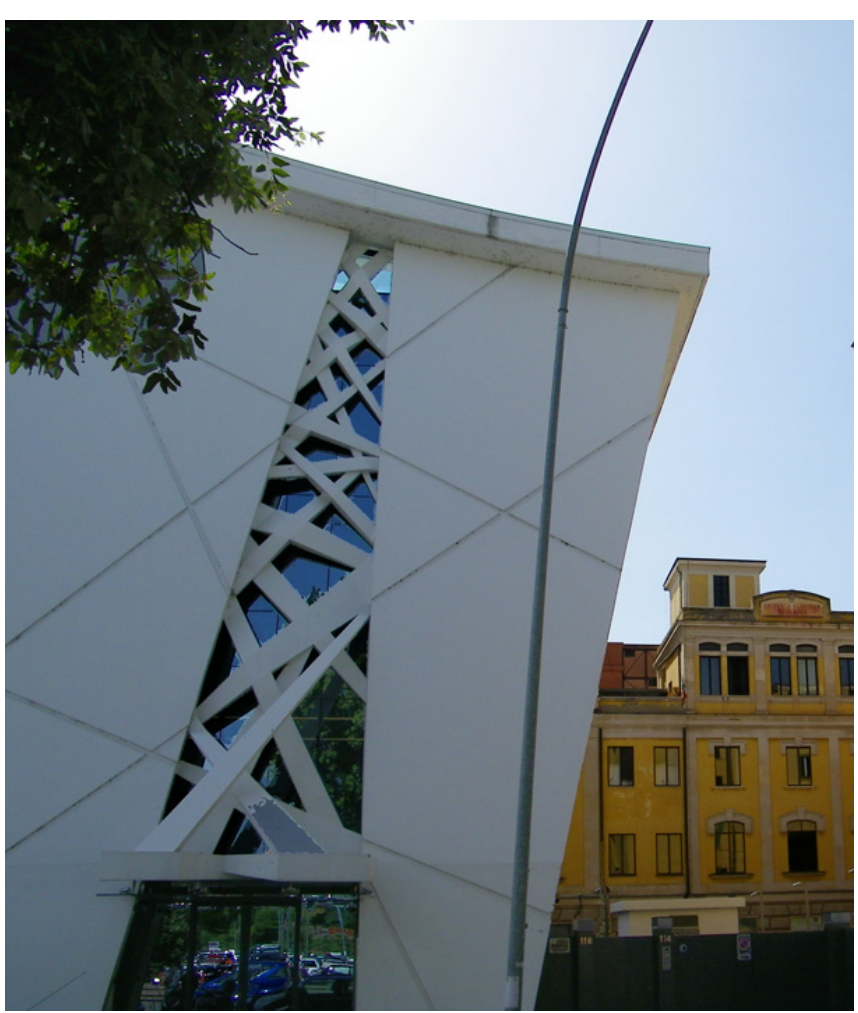

Architecture contemporaine devant l'élévation est de la centrale, via Ostiense. Phot. Buffa, Géraud, 2011. (c) Géraud Buffa. 


\section{BIBLIOGRAPHIE}

BERTOLETTI, Marina, CIMA, Maddalena, TALAMO, Emilia (dir.). Sculture di Roma antica. Milan : Electa, 1999.

FIORE, A. D. La Centrale Termoelettrica Giovanni Montemartini. Dans BERTOLETTI, M., CIMA, M. et TALAMO, E. (dir.). Op. cit.

TORELLI LANDINI, Enrica (dir.). Roma. Memorie della città industriale. Storia e riuso di fabbriche e servizi nei primi quartieri produttivi. Rome : Palombi, 2007.

TORELLI LANDINI, Enrica et TRAVAGLINI, Carlo Maria (dir.). Fabbriche della conoscenza : Roma Tre nel territorio e nella riqualificazione dell'area Ostiense. Catalogue de l'exposition, Rome, rectorat de l'université Rome 3, 20-23 janvier 2001. Rome : Università degli studi Roma Tre, 2001. http:// www.uniroma3.it/downloads/pubblicazioni/catasedirm39900.pdf.

Roma ricerca e formazione. Un patrimonio urbano tra memoria e progetti. Roma : L'area OstienseTestaccio. Catalogue de l'exposition, Rome, Istituto superiore Antincendi, 26 juin-15 octobre 2004. Rome : Università Roma Tre, 2004.

http://www.google.com/url? sa=t\&rct=j\&q=\%22la\%20mostra\%2C\%20attraverso\%20la\%20ricomposizione\%20\%22\&source=web\&cd=1\&ved=0CCMQFjAA\&url=http\%3A\% Site internet : http://fr.centralemontemartini.org/.

\section{NOTES}

1. - L'AEM de Rome, qu'il ne faut pas confondre avec l'AEM de Milan, deviendra en 1945 l'Azienda comunale Elettricità e Acque (ACEA), puis, en 1989, l'Azienda Comunale Energia e Ambiente (ACEA), qui est aujourd'hui encore un important groupe énergétique italien.

2. - Centre de culture scientifique, technique et industrielle.

3. - Le macchine e gli dei. Voir TALAMO, Emilia. Le macchine e gli dei. Sculture antiche nel primo impianto romano di produzione di energi elettrica, lien vers http://aragon.es/estaticos/ GobiernoAragon/Departamentos/EducacionCulturaDeporte/Documentos/docs/Areas/Jornadas/ Jornadas_Patrimonio_Industrial/04\%20Le\%20macchine\%20e\%20gli\%20dei.pdf.

4. - Auteur inconnu, 1891 ; publiée dans HURLEY, F. Jack. Industry and the Photographic Image. Rochester/New York : G. Eastman House/Dover publications, 1980.

5. - Constantin Meunier (1831-1905), peintre et sculpteur réaliste belge.

6. - Lewis Wickes Hine (1874-1940), photographe américain, connu en particulier pour ses photographies d'enfants au travail.

7. - Cette absence se retrouve à la lecture des publications mises à la disposition du public sur cette nouvelle antenne des musées du capitole. Depuis la pérennisation du site, ces publications accordent une place un peu plus importante à l'histoire de la centrale et la description des machines, mais cet aspect n'y occupe malgré tout qu'un chapitre et celui-ci ne permet pas de comprendre les objectifs de la confrontation.

8. - Le MACRO est devenu dès 2003 le MACRO Future et s'est agrandi.

9. - « Centro di produzione culturale ». 


\section{RÉSUMÉS}

Inaugurée en 1912, la centrale électrique de Montemartini fut la première centrale électrique publique de Rome. À partir de 1997 - une trentaine d'années après sa fermeture - une partie des collections de sculpture romaine des musées du Capitole y a été déployée. Cette installation, provisoire à l'origine, a été pérennisée en 2005 grâce au succès public qu'elle a rencontré. Si les tenants et aboutissants de ce succès sont difficiles à analyser, il est indéniable que la centrale est devenue, au cœur du quartier Ostiense-Marconi, l'emblème de la reconquête du patrimoine industriel entreprise par la Ville de Rome.

Opened in 1912, the Montemartini powerplant was the first public power station of Rome. From 1997 (about thirty years after it closed) some collections of Roman sculpture of the Capitoline Museums were displayed there. The exhibition, originally temporary, was made permanent in 2005 thanks to its huge public success. If the ins and outs of this success are difficult to analyze, it is undeniable that the plant has become, in the heart of Ostiense-Marconi area, the symbol of the reconquest of the industrial heritage undertaken by the city of Rome.

\section{INDEX}

Keywords : Rome, industrial heritage, classical sculpture, reconversion, machines, gods, coalfired power station, diesel engine, Montemartini, Ostiense, Tosi

Mots-clés : Rome, patrimoine industriel, sculpture antique, reconversion, machines, dieux, centrale thermique, moteur diesel, Montemartini, Ostiense, Tosi

\section{AUTEUR \\ GÉRAUD BUFFA}

conservateur en chef du patrimoine, chercheur au service de l'Inventaire général et $d u$ patrimoine de la Région Provence-Alpes-Côte d'Azur, gbuffa@regionpaca.fr 